\title{
Do you know the extinction in your young massive cluster?
}

\author{
Guido De Marchi ${ }^{1}$, Nino Panagia ${ }^{2}$ and Elena Sabbi ${ }^{2}$ \\ ${ }^{1}$ ESTEC, 2200 AG Noordwijk, The Netherlands, email: gdemarchi@esa.int \\ ${ }^{2}$ STScI, Baltimore, MD 21218, United States, email: panagia@stsci.edu, sabbi@stsci.edu
}

Up to ages of $\sim 100 \mathrm{Myr}$, massive clusters are still swamped in large amounts of gas and dust, with considerable and uneven levels of extinction. At the same time, large grains (ices?) produced by type II supernovae profoundly alter the interstellar medium (ISM), thus resulting in extinction properties very different from those of the diffuse ISM. To obtain physically meaningful parameters of stars, from basic luminosities and effective temperatures to masses and ages, we must understand and measure the local extinction law. This problem affects all the massive young clusters discussed in his volume.

We have developed a powerful method to unambiguously determine the extinction law in an uniform way across a cluster field, using multi-band photometry of red giant stars belonging to the red clump (RC). In the Large Magellanic Cloud (LMC), with about 20 $\mathrm{RC}$ stars per $\operatorname{arcmin}^{2}$, we can easily derive a solid and self-consistent absolute extinction curve over the entire wavelength range of the photometry. Here, we present the extinction law of the Tarantula nebula (30 Dor) based on thousands of stars observed as part of the Hubble Tarantula Treasury Project (HTTP; Sabbi et al. 2013, 2015). Space limitations only allow us to highlight the main results. For more details see De Marchi et al. (2015).

From the colour-magnitude diagrams (CMD) we can derive the absolute extinction in all bands towards $\sim 3500$ red giants that belong to the RC. The arrows in Fig. 1 indicate the position of the un-reddened $\mathrm{RC}$. The high and variable levels of extinction spread RC stars across the CMD defining tight strips. The slopes of such strips in all bands provide a direct measure of the reddening vector.

In the Tarantula nebula the reddening vector is much steeper than in the Galaxy. While in the diffuse Galactic ISM $R_{V}=A_{V} / E(B-V)=3.1$ (thick blue dashed line in Fig. 2), the Tarantula extinction curve (dots and red line) implies $R_{V}=4.5 \pm 0.2$. This value is in excellent agreement with the measurements made inside its core $\mathrm{R} 136$ both from photometry $\left(R_{V}=4.4 \pm 0.2\right.$, black dashed line; De Marchi \& Panagia 2014) and spectroscopy $\left(R_{V}=4.4 \pm 0.4\right.$; Maíz Apellániz et al. 2014). At optical wavelengths the extinction law is practically parallel to the Galactic law, being shifted to higher values of $R$ by an amount of 0.8 (thin short-dashed line). The difference between the extinction law in 30 Dor and in the Galaxy (green line) is remarkably flat in the optical. This indicates that the extinction curve in the Tarantula is due to dust of the same type as in the Galaxy, but with an extra component that does not depend on wavelength ("grey"). At wavelengths longer than $\sim 1 \mu \mathrm{m}$ the contribution of this grey component tapers off as $\lambda^{-1.5}$, like in the Milky Way (dot-dashed line), further suggesting that the nature of the grains is otherwise similar to those in our Galaxy, but with a $\sim 2$ times higher fraction of large grains. This is consistent with the addition of "fresh" large grains by type II supernova explosions, as recently revealed by the Herschel and ALMA observations of SN 1987A (e.g. Matsuura et al. 2011; Indebetouw et al. 2014).

These results leave no doubt that in the Tarantula large grains are more important than in the diffuse ISM in the Galaxy. These properties are not found in the surroundings of the 

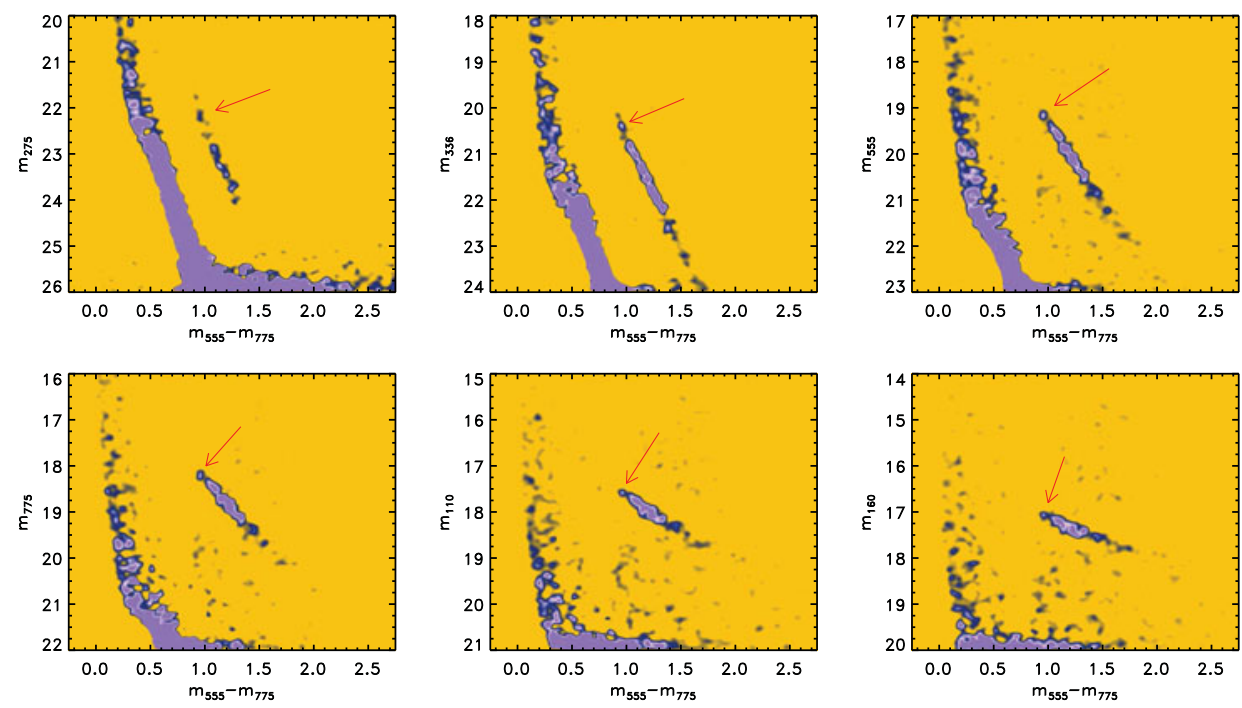

Figure 1. Unsharp masking applied to all CMDs clearly reveals the location of the un-extinguished RC. The reddening vector is obtained through a linear fit to the elongated RC.

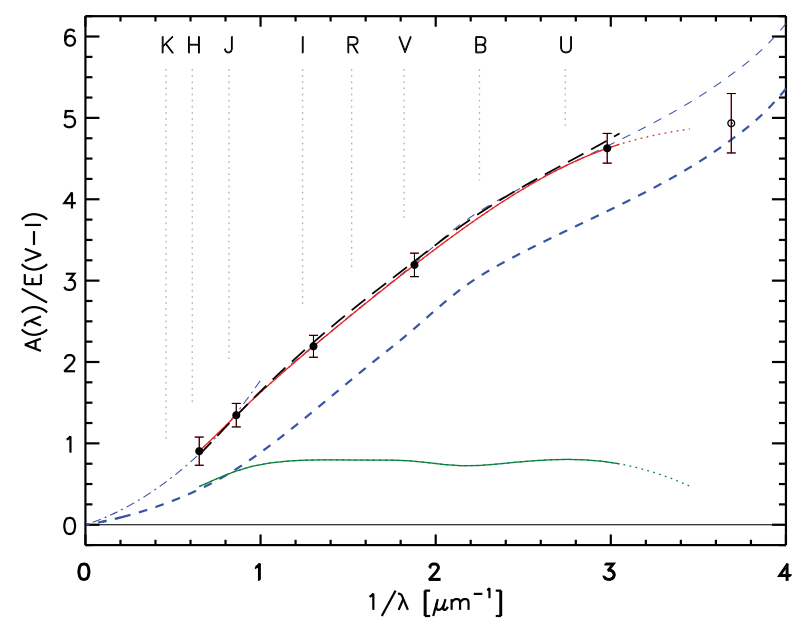

Figure 2. The extinction law of the Tarantula nebula (dots and red line) is compared with the one measured inside the nebula's core (R 136; long-dashed line), and with that of the diffuse Galactic ISM (thick short-dashed line). In the optical domain, the Tarantula and Galactic curves are parallel (the green line is their difference). See the text for details.

Tarantula, so they must result from changes related to the recent/ongoing star formation, implying that the ISM is dynamically altered as large fresh grains are produced and later destroyed. These findings have important implications for the study of the star formation properties in galaxies. Even though these changes might last only some $\sim 50 \mathrm{Myr}$, they affect the HII regions at the peak of their luminoisty, when they are most easily detectable in distant galaxies. Therefore, assuming typical ISM conditions could result in severely underestimated total masses and star formation rates, by a factor of 2 or more.

\section{References}

De Marchi, G. \& Panagia, N. 2014, MNRAS, 445, 93

De Marchi, G., Panagia, N., Sabbi, E., et al. 2015, MNRAS, submitted

Indebetouw, R., et al. 2014, ApJ, 782, L2

Maíz Apellániz, J., et al. 2014, A\&A, 564, A63

Matsuura, M., et al. 2011, Sci, 333, 1258

Sabbi, E., et al. 2013, AJ, 146, 53

Sabbi, E., et al. 2015, ApJS, submitted 